Recherches en histoire de l'art, histoire des civilisations, archéologie, anthropologie et muséologie

\title{
Lumière sur Eugène Martial Simas, décorateur oublié de la Belle Époque
}

\section{Constance Desanti}

\section{OpenEdition}

\section{Journals}

Édition électronique

URL : http://journals.openedition.org/cel/289

DOI : $10.4000 /$ cel.289

ISSN : 2262-208X

Éditeur

École du Louvre

\section{Référence électronique}

Constance Desanti, « Lumière sur Eugène Martial Simas, décorateur oublié de la Belle Époque », Les Cahiers de l'École du Louvre [En ligne], 7| 2015, mis en ligne le 01 octobre 2015, consulté le 17 septembre 2019. URL : http://journals.openedition.org/cel/289; DOI : 10.4000/cel.289

\section{(2) $(\mathcal{Q} \Theta \Theta$}

Les Cahiers de l'École du Louvre sont mis à disposition selon les termes de la licence Creative Commons Attribution - Pas d'Utilisation Commerciale - Pas de Modification 4.0 International. 


\section{Cahiers de l'École du Louvre}

recherches en histoire de l'art, histoire des civilisations archéologie, anthropologie et muséologie

Numéro 7. Octobre 2015

Lumière sur Eugène Martial Simas, décorateur oublié de la Belle Époque Constance Desanti

Article disponible en ligne à l'adresse :

http://www.ecoledulouvre.fr/cahiers-de-l-ecole-du-louvre/numero7octobre-2015/desanti.pdf

Pour citer cet article :

Constance Desanti, « Lumière sur Eugène Martial Simas, décorateur oublié de la Belle Époque »[en ligne] n 7, octobre 2015, p. 64 à 75.

\section{@creative \\ $\circledast \circledast$}

(c) École du Louvre

Cet article est mis à disposition selon les termes de la Licence Creative Commons Attribution - Pas d'utilisation commerciale - Pas de modification 3.0 non transposé. 


\section{Cahiers de l'École du Louvre recherches en histoire de l'art, histoire des civilisations archéologie, anthropologie et muséologie}

\section{Numéro 7. Octobre 2015}

\section{Sommaire}

\section{Éditorial}

\section{Étude}

Caroline van Eck, Miguel John Versluys, Pieter ter Keurs, Leiden University, Departments of Art History, Archaeology, Anthropology/National Museum of Antiquities (résumé et texte intégral en pdf)

The biography of cultures: style, objects and agency. Proposal for an interdisciplinary approach.

\section{Articles}

Camille Bourdiel, ancienne élève de l'École du Louvre, diplômée de $2^{\mathrm{e}}$ cycle (résumé et texte intégral en pdf)

Exposer la science dans l'après-guerre. Hommage à Léonard de Vinci et Rembrandt, étude photographique et radiographique

au laboratoire du musée du Louvre.

Fernando Suárez San Pablo, ancien élève de l'École du Louvre, diplômé de $2^{\mathrm{e}}$ cycle (résumé et texte intégral en pdf)

La politique de restauration

des peintures des musées nationaux (1930-1950).

Alexandra Buvignier-Legros, ancienne élève de l'École du Louvre, diplômée de $2^{\mathrm{e}}$ cycle (résumé et texte intégral en pdf)

Pascal Häusermann et le motel L'Eau vive : une conception d'avant-garde ?... p. 46-53

Agnès Gué, ancienne élève de l'École du Louvre, diplômée de $2^{\mathrm{e}}$ cycle (résumé et texte intégral en pdf)

Goya dans l'historiographie française du XIX siècle : images et textes.

p. $54-63$

Constance Desanti, ancienne élève de l'École du Louvre, diplômée de $2^{\mathrm{e}}$ cycle (résumé et texte intégral en pdf)

Lumière sur Eugène Martial Simas, décorateur oublié de la Belle Époque. ...p. 64-75

Clémentine Delplancq, ancienne élève de l'École du Louvre,

diplômée de $2^{\mathrm{e}}$ cycle (résumé et texte intégral en pdf)

Faire carrière à Paris : Armand Bloch (1866-1932) et la Franche-Comté,

l'importance du soutien régional pour les artistes au XIX siècle.

p. $76-85$

Claire Merleau-Ponty, consultante en muséologie, (résumé et texte intégral en pdf) Vous avez dit médiation?

p. $86-88$

Andréa Delaplace, ancienne élève de l'EHESS, diplômée de $2^{\mathrm{e}}$ cycle (résumé et texte intégral en pdf)

Un palais pour les immigrés? Le Musée de l'histoire de l'immigration à Paris :

une collection et un musée en devenir.

p. $89-99$

Joan Despéramont, ancienne élève de l'École du Louvre, diplômée de $2^{\text {e }}$ cycle (résumé et texte intégral en pdf)

Les Souffleurs d'images. Un concept développé

par le Centre de Recherche Théâtre-Handicap (CRTH).

p. $100-106$ 
Constance Desanti

Décorateur, peintre et illustrateur, Eugène Martial Simas (1862-1939) fut à l'origine d'une œuvre féconde largement saluée par les critiques de son temps. Pourtant, si l'on peut encore de nos jours contempler ses réalisations dans les gares, brasseries ou demeures privées, son nom a quelque peu disparu de la mémoire collective. C'est à la lumière de deux récents projets de restauration de compositions dont il est l'auteur - les vitraux laïcs de la demeure Art nouveau de Belle-Île, dite " château Laurens ", à Agde (Hérault) et les panneaux en céramique émaillée de la gare de Tours - qu'il apparaît propice d'éclairer d'un jour nouveau cet artiste si singulièrement ancré dans notre patrimoine.

\section{Des débuts prometteurs}

Eugène Martial Simas voit le jour le 31 août 1862 à Paris ${ }^{1}$, en pleine révolution industrielle. La ville de Poissy dans les Yvelines, où il grandit ${ }^{2}$ avec son frère cadet, abrite alors la fabrique de chapeaux familiale que créa en 1853 son grand-père maternel, Gabriel Gibus, célèbre inventeur du chapeau claque. Cependant, bien qu'il fût probablement imprégné par l'univers artisanal et bientôt mécanisé de la chapellerie, ce n'est pas vers ce champ d'activité que le jeune Eugène Martial se tourne à ses débuts, mais vers la conception de décors de théâtre. C'est en effet auprès du peintre en décor Jean-Baptiste Lavastre ${ }^{3}$ (1839-1891), lui-même élève d'Édouard Despléchin (1802-1871), grand décorateur de théâtre de la période romantique, que E. M. Simas entame sa formation artistique.

La maîtrise affinée du processus de création que requiert son activité de décorateur de théâtre - travail d'esquisse, réalisation de maquettes puis peinture du décor sur d'immenses toiles tendues - le mène progressivement à élargir son domaine d'application à la décoration de bâtiments publics tels que les mairies.

Ainsi, Simas prend part en 1892 aux concours ouverts visant à orner la salle à manger et les salons d'introduction du nouvel Hôtel de Ville de Paris, rebâti entièrement à la suite des évènements de la Commune. Le Petit Palais, musée des Beaux-Arts de Paris, conserve dans ses réserves les cinq esquisses exécutées par l'artiste à cet effet. Répondant à une commande spécifique de la Ville, ces réalisations illustrent, à l'aide de gracieuses allégories républicaines et religieuses, la vie quotidienne parisienne et clament le triomphe et la renommée de la capitale française. Véritable retranscription iconographique des préceptes moraux incarnés par la III ${ }^{e}$ République, les panneaux vantent alternativement les bienfaits de la révolution industrielle florissante (esquisse Art, Science, Industrie, Commerce) et l'utilité des travaux des champs (esquisse Les Champs, Le Verger, Le Parterre, Le Potager, etc.)

Si Eugène Martial Simas ne remporte pas ces deux concours, décrochés par Georges Bertrand et Bonis, ce « coup d'essai » lui permet de se faire remarquer et

\footnotetext{
1. Archives en ligne de la ville de Paris, acte de naissance d'Eugène Martial Simas daté du $1^{\text {er }}$ septembre 1862 : «Du premier septembre mil huit cent soixante-deux, dix heure du matin acte de naissance de Eugène Martial [...] né le trente et un août dernier [...] rue Beaubourg $n^{\circ} 40$, fils de Louis Émile Simas, fabricant de chapeaux, âgé de quarante ans et de Catherine, Adolphine Victorine Gibus, sans profession, âgé de trente et un ans ».

2. Le Manuel Général de l'instruction primaire, n 35, du 29 août 1874, nous apprend qu'Eugène Martial Simas a été élève à l'école libre de Poissy (Seine et Oise), alors dirigée par M. Duplessier. 3. Anonyme, «Derrière la toile», Le Rappel, 24 janvier 1894: « décor artistique de M. Martial Simas élève de J. B. Lavastre ».
} 
d'obtenir cinq ans plus tard la commande du décor de la salle des mariages de la mairie de Créteil ${ }^{4}$.

Au cœur de cet édifice élevé au XviII ${ }^{\mathrm{e}}$ siècle par Nicolas le Camus de Mézières, les quatre toiles marouflées signées de Simas viennent revêtir la pièce de l'union civile de paysages rustiques de Créteil et de ses environs. Il faut en effet garder à l'esprit qu'en 1897, Créteil n'est alors qu'une petite bourgade rurale qui vit au rythme des saisons, des semis et des moissons. Dans un style naturaliste et réaliste proche de celui des peintres de l'École de Barbizon tels François Daubigny ou Jean-François Millet, Eugène Martial Simas dépeint la vie quotidienne de Créteil et de ses habitants : canotage et promenade dominicale en famille au bord de la Marne, labourage des champs, coupe du bois en forêt au seuil de l'hiver ou encore déambulation de cyclistes et de voitures tractées par des chevaux sur l'artère principale de la ville.

Une fois n'est pas coutume, la thématique privilégiée est celle d'un idéal républicain célébrant l'amour heureux et la famille, mais aussi le travail qui est, par la productivité, le soutien à la nation. Cela n'a d'ailleurs rien d'étonnant car rappelons que dans l'optique de la rentabilité économique du XIX ${ }^{e}$ siècle, mariage et travail étaient liés. Dans une grande attention au détail, Simas parfait cette décoration de lambris peints de bouquets de lys et de lauriers symbolisant la pureté et la réussite dans le mariage.

\section{De la peinture monumentale aux arts décoratifs et industriels}

Eugène Martial Simas aurait pu se cantonner à son activité de peintre en décor, mais, en observateur attentif des bouleversements sociaux et des avancées techniques et scientifiques que le pays connaît alors, il voit plus loin. Durant la décennie 1890, il décide de donner une nouvelle tournure à sa carrière de décorateur, en s'orientant vers les arts appliqués à l'industrie. Les supports d'application de ses créations sont alors extrêmement diversifiés, allant de la porcelaine de Sèvres au vitrail coloré en passant par le mobilier d'intérieur, les tissus brodés, les carreaux de faïence fine de Sarreguemines, le papier et le métal chromolithographiés. L’œuvre finale résulte donc de l'association essentielle entre son pinceau d'artiste et le savoir-faire de l'artisan ou la technique de l'industriel. Cette démarche créatrice collective d'un genre nouveau est portée aux nues par le critique d'art Roger Marx dans son ouvrage manifeste L'Art social (1913) où il avance : " Un abus fréquent met en conflit le travail à la main et le travail à la machine ; ils peuvent, ils doivent coexister [...] Leur pratique simultanée est indispensable. " 5 Dans cette lignée, l'œuvre de Simas incarne parfaitement cette volonté de renouvellement des moyens plastiques à l'aube $\mathrm{du} \mathrm{Xx}^{\mathrm{e}}$ siècle qui conduira progressivement à la remise en cause de la hiérarchie des genres et à l'affirmation de la place prépondérante des arts décoratifs et industriels dans la société moderne.

\section{Une collaboration fructueuse avec la manufacture de Sarreguemines}

En 1895, alors âgé de 33 ans, Eugène Martial Simas entreprend de travailler avec la manufacture alsacienne de Sarreguemines. La fabrique, créée au lendemain de la Révolution française par trois négociants, s'agrandit rapidement et fonde des succursales à Digoin, en 1877 et à Vitry-le-François, en 1881. Réputée pour la blancheur et la finesse de son biscuit, la faïence qui y est produite devient très prisée et se répand largement à la suite de l'invention du chemin de fer. Simas conçoit de nombreux modèles, diffusés grâce aux catalogues de vente que la manufacture édite, qui seront ensuite fabriqués sous la forme de panneaux carrelés

4. Archives de la mairie de Créteil, dossier sur la maison du combattant, document D1/14, délibération du conseil municipal de Créteil, texte du préfet devant le conseil général de la Seine, le 23 octobre 1897 : «Je vous proposerai de confier la décoration de la mairie de Créteil à M. Eugène Martial Simas, jeune artiste de talent dont les paysages ont été remarqués au Salon du Champ de Mars et qui a été primé au concours ouvert pour la décoration de la salle à manger de I'hôtel de ville de Paris ».

5. Roger Marx, L'Art social, Paris, éditions E. Fasquelle, 1913, p. 12. 
de faïence, recouverts d'émaux cloisonnés à l'engobe. La préciosité des matériaux utilisés et la grande qualité d'exécution de ces créations témoignent du savoirfaire de la manufacture qui contribue à la réalisation d'œuvres d'art à part entière.

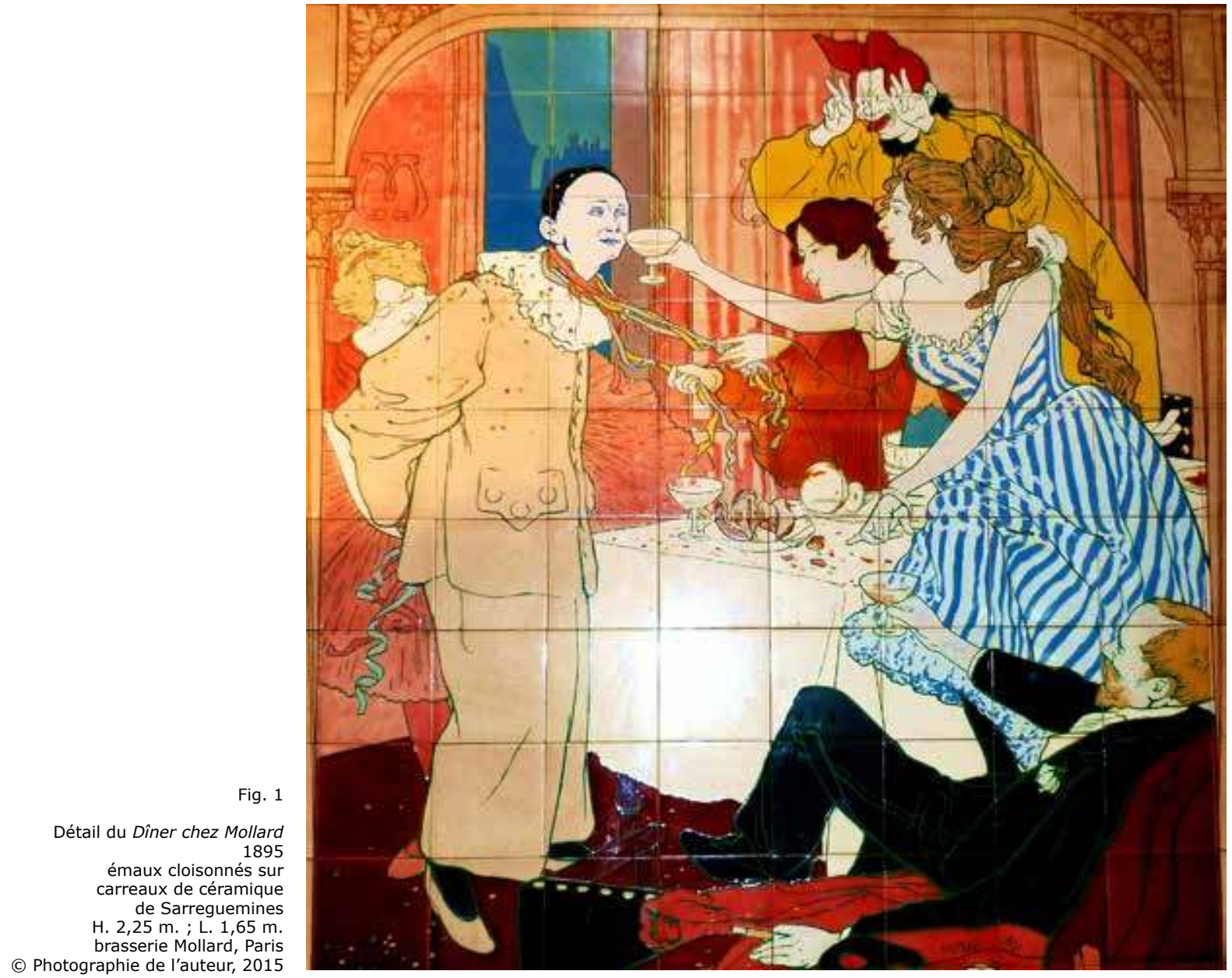

À la suite d'une réflexion globale sur la salubrité publique, de nombreux « lieux d'hygiène " se dotent de décors de faïence qui présentent l'avantage de se nettoyer facilement. Parmi les commanditaires de ces tableaux brillants, on trouve les cafés. La brasserie Mollard est l'incarnation même de cette volonté d'assainir la ville à l'approche de l'Exposition universelle de 1900. Situé dans le quartier de la gare Saint-Lazare à Paris, l'édifice dont on doit l'aménagement intérieur à l'architecte Édouard-Jean Niermans (1859-1928) devient « le rendez-vous des voyageurs du monde entier ${ }^{6} »$. Dans ce décor fastueux composé de mosaïques or de la maison Bicchi et de marbres précieux, prennent place neuf panneaux de Sarreguemines signés de la main de Simas. Dans le premier salon en entrant, deux panneaux illustrent l'arrivée et le départ de la gare d'une jeune femme. Vêtue dans l'exacte tendance de la mode féminine des années 1895-1900 avec son chapeau garni de nœuds et sa robe dotée de manches gigot, elle descend les marches de la gare saint-Lazare toute proche pour se rendre à la capitale avant de regagner la banlieue le soir venu, les bras chargés de paquets. Plus loin, trois autres panneaux s'offrent à notre vue. Sur le thème identique de la gare saint-Lazare et des plaisirs que permettent les villes qu'elle dessert, ils plaident tour à tour les vertus apaisantes du canotage à Saint-Germain-en-Laye, les bienfaits des bains de mer d'une station balnéaire comme Trouville et la fraîcheur de la campagne à Ville-d'Avray. Ce sont aussi deux triptyques - dont l'un a malheureusement disparu et qui autrefois se faisaient face - dépeignant les festivités de la table. Le premier (fig. 1), encadré par deux allégories de l'Automne et de l'Hiver, fait flamboyer sous les cotillons et

6. L. Couturat, «L'architecture moderne, une brasserie modèle », Gil Blas (supplément hebdomadaire du samedi), juin 1896. 
les confettis, l'ivresse d'une soirée parisienne où de jeunes noceurs grisés, incarnés entre autre par un clown diabolique et une danseuse en tutu, tentent de faire oublier le chagrin d'un pierrot mélancolique.

Quant au second, se plaçant sous les auspices du Printemps et de l'Été, il dévoilait le déjeuner en plein air de convives endimanchés dont une demoiselle arborant une robe aux motifs japonisants. Le décor de la brasserie s'achève enfin par des allégories de l'Alsace et de la Lorraine, reflet probable de la nostalgie causée par la perte récente de ces deux régions françaises.

L'année 1895 correspond également à la décoration par Eugène Martial Simas de deux autres panneaux de fä̈ence de Sarreguemines ornant la brasserie La Cigale, à Nantes. CEuvre d'Émile Libaudière (1853-1923), l'édifice à l'architecture de fer apparente présente lui aussi un décor riche et coloré, agrémenté de peintures de Georges Levreau (1867-1936) et de sculptures d'Émile Gaucher (1860-1909). Dans ce lieu dédié à la boisson, Simas met en scène deux jeunes femmes, l'une vêtue d'une robe rose printanière portant à ses lèvres un bock de bière et l'autre, à l'ondulation serpentine, brandissant une coupe de champagne mousseux qui s'écoule lentement à la lueur des lampions japonais.

Notons enfin que deux autres panneaux de l'artiste figurent cette fois-ci sur le perron d'un restaurant parisien, Le Petit Zinc - anciennement connu sous le nom de L'Assiette au beurre - niché au cœur du Quartier Latin. Incorporés à la façade céladon de l'édifice, modèle Art nouveau rappelant les bouches de métro d'Hector Guimard, ils invitent l'éventuel client à s'immerger dans une atmosphère bucolique suggérée par une jeune Lorraine cueillant des iris jaunes et par une Bressane récoltant les œufs du poulailler d'un jardin potager.

Trois ans plus tard, de nouveaux panneaux signés Eugène Martial Simas sont commandés à la manufacture pour décorer un tout autre lieu, la gare de Tours, fraîchement érigée par l'architecte Victor Laloux (1850-1937), également auteur de la gare d'Orsay. Le bâtiment, inscrit au titre des monuments historiques depuis 1984, est un chef-d'œuvre d'architecture de pierre, de verre et de fer au sein duquel les panneaux de Simas prennent toute leur dimension. Parmi les seize pièces installées, neuf sont signées de l'artiste? ${ }^{7}$. Véritable invitation au voyage et à la détente, ces compositions brillantes représentent chacune une destination que la gare dessert. Flanc de colline parsemé d'arbres et de rochers pour Vic-surCère, cité fortifiée où ondulent de petits chats pour Carcassonne, longs pins se balançant au vent pour Arcachon, petits ruisseaux rocailleux dominés par le massif auvergnat pour le Mont-Dore et ainsi de suite jusqu'au neuvième panneau qui dépeint Saint-Jean-de-Luz, station balnéaire du Pays Basque où deux promeneuses contemplent l'océan du haut des falaises. Ce dernier panneau est un des plus évocateurs par son style japonisant qui met en contraste le bleu de Prusse de l'eau et le blanc nacré de la roche calcaire si caractéristique du littoral basque.

Ajoutons qu'en plus de leur fonction décorative, ces "cartes postales" monumentales, par leur séduisante mise en scène, représentaient un moyen non négligeable pour les compagnies de chemin de fer d'élargir leur clientèle, sensible à cette nouvelle forme de publicité touristique.

\section{Un encas savoureux : la rencontre du biscuit émaillé avec le petit-beurre nantais}

La participation de Simas au décor de La Cigale attire l'attention de l'industriel nantais Louis Lefèvre-Utile qui lui commande en 1896, par l'entremise de la manufacture de Sarreguemines, un ensemble de panneaux décoratifs pour orner son nouveau siège. Héritier de la pâtisserie familiale en 1883, Louis Lefèvre-Utile entend bien agrandir la petite entreprise et rendre visible l'image de la marque. Pour ce faire, il rachète une ancienne filature dont les bâtiments bordent l'un

7. Parmi les seize panneaux muraux de la gare de Tours dépeignant les sites de Touraine, de Bretagne, d'Auvergne et du sud-ouest, les neuf que I'on doit à $E$. M. Simas sont : Vic-sur-Cère, Carcassonne, Arcachon, Pont de Cahors, Mont-Dore, Amboise, Luchon, Fontarabie, Saint-Jeande-Luz. 
des principaux bras de la Loire et dont les machines fonctionnent à la vapeur. Il s'entoure également des meilleurs illustrateurs de son époque. Mettre l'art au service de la consommation est en effet indispensable pour cet entrepreneur qui souhaite se façonner une image de marque répondant à tous les raffinements de l'époque.

Aussi, après avoir approché le célèbre affichiste tchèque Alfons Mucha, il suggère à Eugène Martial Simas deux compositions autour des thématiques du sport et du Teatime ${ }^{8}$, à partir desquelles naîtront les panneaux Le Samovar et Le Tandem, placés dans l'escalier d'honneur et dans la cour intérieure du siège de la maison LU. Un an plus tard, Simas réalise un plateau circulaire pour la présentation du gâteau nantais ${ }^{9}$ (fig. 2 ) puis de petits seaux à biscuits en forme de bonbonnière imprimés sur plaques de fer blanc.

Fig. 2

à gâteau nantais Le goûter sur I'herbe gouache, aquarelle et encre sur papier Collection Familiale Lefèvre-Utile-Fruneau, photo Jehan Romé, courtesy Olivier Fruneau

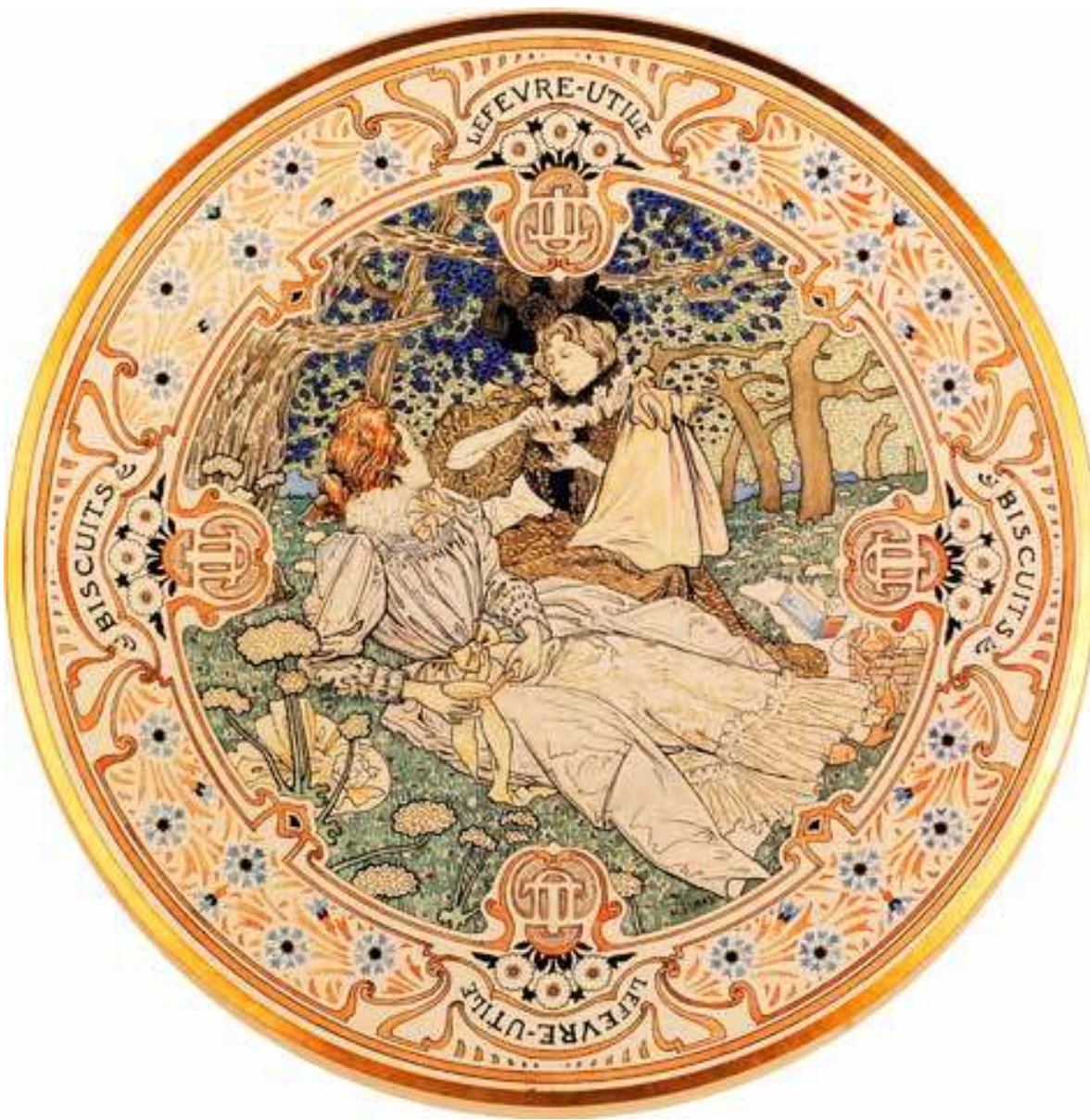

Les deux premiers modèles, les Souris et les Coquelicots, charmantes compositions scandées d'épis de blé rappelant la fabrication du petit-beurre, sont imprimés par Alfred Riom. Les deux suivants, les Chats et les Bleuets sont quant à eux, imprimés par Champenois. La dernière création d'E.M. Simas pour LU, datant de 1903, est une boîte en fer blanc rectangulaire illustrant la récréation d'enfants qui se régalent de biscuits de la marque ${ }^{10}$.

On doit enfin à Simas le dessin du monogramme emblématique de la maison LU trônant au-dessus des deux tours de l'usine nantaise élevées en 1909 par l'architecte parisien Auguste Bluysen (1868-1952).

8. Fonds Lefèvre-Utile, archives départementales de Loire Atlantique, Nantes, correspondance entre Louis Lefèvre-Utile et Eugène Martial Simas : cartons 118 J8-118 j9

9. Fonds Lefèvre-Utile, idem : cartons 118 J9-118 J10

10. Au sujet des créations d’Eugène Martial Simas pour LU, et de manière générale, pour approfondir l'histoire des différents conditionnements de la célèbre marque de biscuits, nous renvoyons à la lecture de I'ouvrage richement illustré d'Olivier Fruneau-Maigret, LU, un siècle d'habillages et de boîtes mythiques, La Ferté-Bernard, éditions Daniel Bordet, 2010. 


\section{Verrières arc-en-ciel et salle de bain végétale : les décors vivifiants du Château Laurens}

Poursuivant son travail de dessinateur pour l'artisanat et l'industrie, E.M. Simas s'associe dès 1896 au peintre-verrier Théophile Laumonnerie (1863-1924) dont il se fait le cartonnier ${ }^{11}$. Deux ans plus tard, les deux hommes sont réunis par Emmanuel Laurens pour le projet de décoration de sa surprenante villa se dressant sur le domaine de Belle-Île, bordé par le fleuve Hérault. Le propriétaire leur passe commande de cinq verrières afin de décorer les petits-appartements de sa bâtisse ${ }^{12}$. Si l'association des deux hommes est fructueuse sur le plan artistique, il semble qu'elle l'ait aussi été sur le plan humain puisque Théophile Laumonnerie devient le témoin de mariage de Simas la même année ${ }^{13}$.
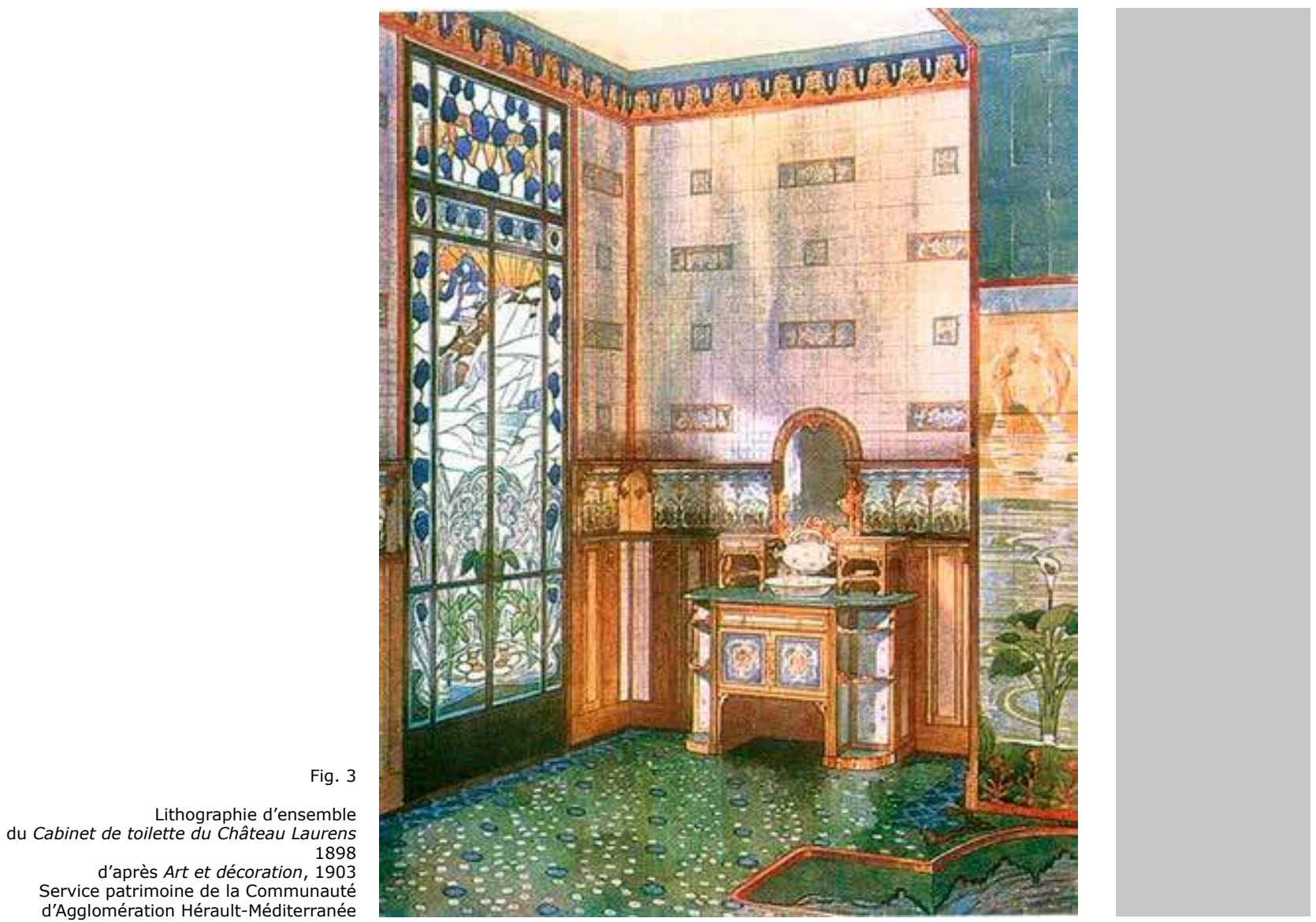

La plus imposante des verrières, $\mathrm{La}$ Mer, décorait le salon-bureau d'Emmanuel Laurens. Représentant un grand paysage marin bordé par des falaises, elle figure en son centre une sirène qu'implore une jeune femme accompagnée d'un enfant. Le traitement des ondes marines n'est pas sans rappeler le japonisme véhiculé en Europe par l'estampe et, dans le cas présent, par la célèbre Vague d'Hokusai (17601849). La touche poétique du vitrail est enfin sublimée par un cartouche placé à l'étage inférieur et renfermant les vers du poète parnassien Eugène Le Mouël : "Sirènes de mer au sourire d'écume, aux yeux verts, aux cheveux que dorent les couchants, votre charme est mortel! Pour vos palis de brume, vous tentez

11. Dès 1896, Eugène Martial Simas et Théophile Laumonnerie exposent le vitrail La Mer au salon de la société nationale des Beaux-Arts et au salon du Champ de Mars.

12. Nous devons à Laurent Félix chargé du patrimoine et de la villa Laurens pour la Communauté d'Agglomération Hérault Méditerranée, un article approfondi sur les décors du Château Laurens. Intitulé « Le château Laurens et son décor : une demeure remarquable à Agde en 1900 » et paru en 2013 dans les Études héraultaises, il retrace I'histoire de la villa, revient sur les artistes qui I'ont façonnée et fait le point sur les phases de restauration réalisées.

13. Archives en ligne de la ville de Paris, acte de mariage d'Eugène Martial Simas avec Anne Marie Dalbergue, signé le 3 novembre 1898 dans le $18^{\mathrm{e}}$ arrondissement de Paris. 
d'arracher les hommes à leurs champs, et vous perdez les fils des femmes par vos chants. Sirènes de la mer au sourire d'écume ${ }^{14}$."

Mais le véritable chef-d'œuvre qu'Eugène Martial Simas réalise pour la demeure d'Emmanuel Laurens est sans nul doute une salle de bain, projet auquel prennent part avec lui toute une équipe d'artistes et d'artisans : Gian Domenico Facchina réalise le sol fleuri en mosaïque de marbre et d'émail, Théophile Laumonnerie peint le vitrail chenillé d'un glacier, quant à Alexandre Charpentier et Félix Aubert, membres du mouvement L'Art dans Tout, ils conçoivent les modèles des baigneuses et des plantes aquatiques qui animent les revêtements muraux en faïence de Sarreguemines. Sur le thème de l'eau et de la nature sauvage, la pièce qu'imagine Simas dans son entièreté développe un programme iconographique d'une grande cohérence. L'eau agit en effet comme le fil conducteur de la pièce : partant du glacier figuré sur le vitrail, elle s'écoule lentement sur le parterre de prairie avant de finir sa course dans le bassin-baignoire où des nénuphars s'entrecroisent. Aussi, les éléments qui constituent ce décor s'accordent à la perfection, formant un ensemble harmonieux dont l'originalité et la délicatesse seront largement salués par la critique et relayés dans les journaux d'art tels que Art et décoration ${ }^{15}$ (fig. 3) et The Studio ${ }^{16}$.

\section{Le lustre de la porcelaine de Sèvres}

Tandis qu'il commence à se forger une renommée certaine, la manufacture nationale de Sèvres, qui connaît alors un rayonnement européen, passe commande à Simas d'une dizaine de modèles de vases à destination de l'esplanade des Invalides à l'approche de l'Exposition universelle de 1900. L'artiste effectue pour cette occasion des compositions originales, dont un vase de taille monumentale intitulée Les Poésies qui développe sur son pourtour une mystérieuse épopée d'inspiration mythologique et médiévale ${ }^{17}$. Ses créations, qui ont le mérite de s'affranchir des modèles anciens, se parent de végétaux stylisés aux notes japonisantes (boutons d'or, trèfles à quatre feuilles, berces des prés) et d'animaux comme ce chat blotti dans un champ de narcisses, animal totémique de son œuvre comme de celle de nombreux autres artistes - on pense à Steinlen ou Bonnard - et qui selon Théodore de Banville, est inséparable du poète tant il incarne «la rêverie, le rythme visible, la Pensée agile et mystique ${ }^{18}$. " Le cadre de l'Exposition universelle accorde une visibilité sans pareille à cette collection réalisée en collaboration avec les céramistes Pihan, Drouet et Gébleux, lui permettant d'être à nouveau présentée au public lors de l'Exposition française d'art décoratif se déroulant à Copenhague en $1909^{19}$.

\section{La nature comme source d'inspiration \\ d'un mobilier « moderne »}

Lors de l'Exposition universelle de 1900, Eugène Martial Simas n'expose pas uniquement avec la manufacture de Sèvres mais rejoint également le pavillon Lefêvre-Utile ainsi que le " groupe XII » dédié au mobilier. Dans cette dernière catégorie, il conçoit l'ensemble d'un hall-bibliothèque pour la maison Jansen, grande firme de décoration fondée en 1880 par le hollandais Jean-Henri Jansen. La pièce qu'il aménage de bout en bout arbore le motif général de la flore et de la forêt. Dans cette «boîte » ouverte au public et recouverte entièrement de lambris en bois de poirier, se love une cheminée de Jean Escoula formée de deux

$\overline{14 . ~ N o u s ~ n ' a v o n s ~ p a s ~ r e t r o u v e ́ ~ l a ~ s o u r c e ~ d u ~ p o e ̀ m e . ~ E u g e ̀ n e ~ L e ~ M o u e ̈ l ~ e ́ t a n t ~ u n ~ c o n t e m p o r a i n ~ e t ~}$ ami de Simas il est possible que ces vers aient été écrits spécialement pour le vitrail.

15. Maurice Guillemot, «Un cabinet de toilette », Art et décoration, juillet-décembre 1903, pp. 399,400 .

16. Anonyme, «A modern bath-room designed by E. M. Simas », The Studio, n 75, juin 1899.

17. Le vase de Beauvais dit Les Poésies est actuellement exposé dans les collections permanentes de la Cité de la céramique, à Sèvres.

18. Théodore de Banville, Le Chat, 1882.

19. Gustave-Roger Sandoz et Jean Guiffrey, Exposition française d'art décoratif de Copenhague, 1909 : rapport général : précédé d'une étude sur les arts appliqués et industries d'art aux expositions, Paris, Comité français des expositions à l'étranger, 1913, p. 94. 
atlantes représentant des travailleurs de la forêt. De nombreux meubles de style Art nouveau viennent garnir l'espace : bureau en bois de violette chaussé de pieds d'angélique et de chèvrefeuille, fauteuils recouverts de velours décorés de primevères, écran à cheminée sculpté de clématites sauvages, etc. ${ }^{20}$ En rupture avec l'éclectisme qui domine alors l'Exposition, Simas prend part, avec d'autres artistes tels que Louis Majorelle, Émile Gallé ou Georges de Feure, au renouveau stylistique de l'ébénisterie française au tournant du siècle.

Poursuivant sur cette lancée, il dessine deux ans plus tard un nouvel ensemble mobilier réalisé par l'ébéniste Paul-Alexandre Dumas pour la maison Barbedienne ${ }^{21}$ (fig. 4). Il est fort probable que les deux hommes se soient rencontrés dans les allées de l'Exposition universelle, Dumas ayant reçu à cette occasion une médaille d'argent pour la présentation d'un intérieur de bibliothèque en acajou sur le thème de l'angélique. Présenté au salon du mobilier de 1902, l'ensemble composé d'un buffet, de deux dressoirs, d'une table et de chaises se décline autour de la plante arbustive du sorbier caractérisée par de minces feuilles oblongues et par de petits fruits rouges.
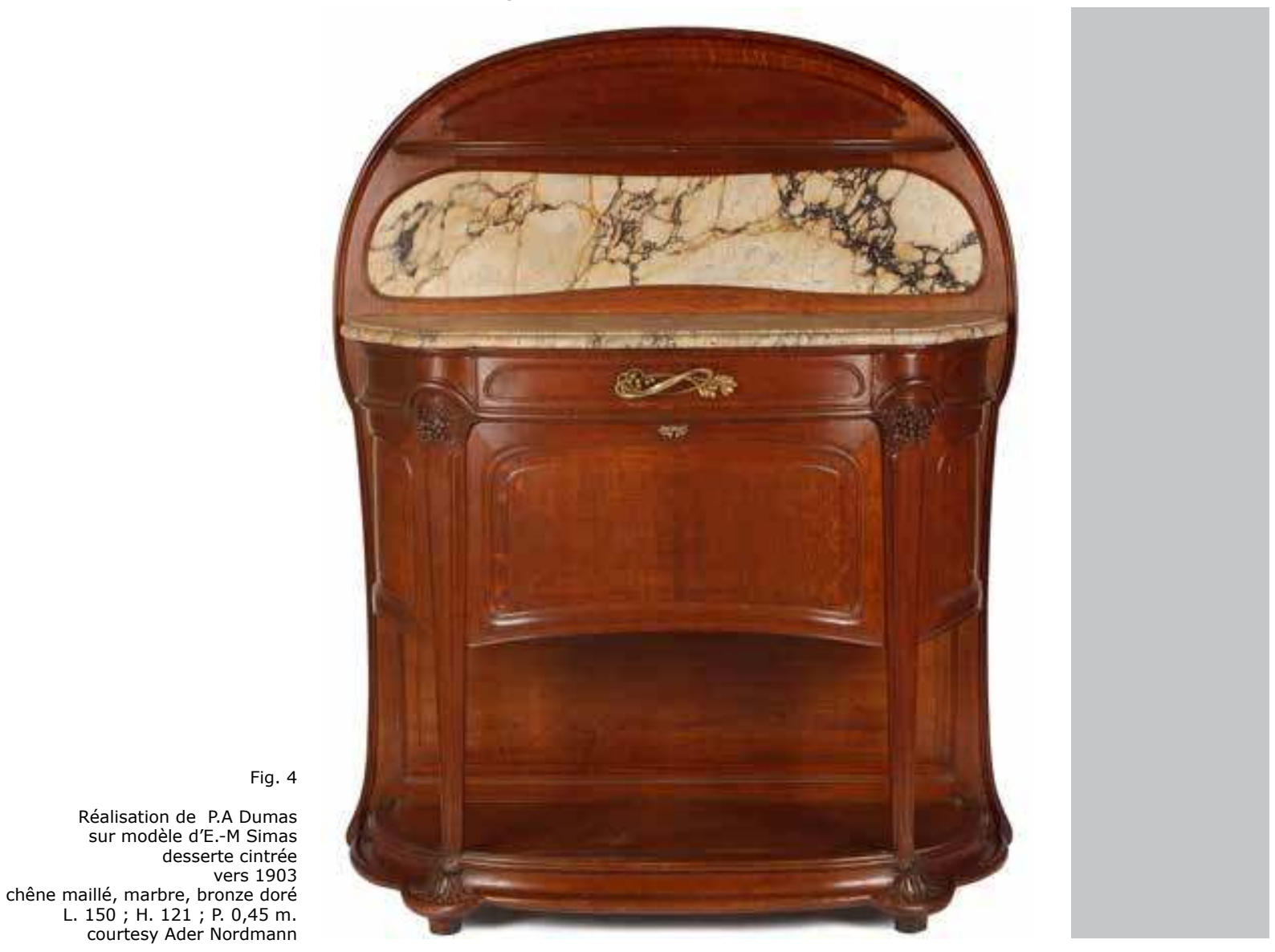

Le style adopté - motifs dérivés de la nature, courbes sinueuses, emploi de matériaux d'origine végétale et minérale tels que le marbre veiné et les bois tropicaux - témoigne de la quintessence de l'Art nouveau qui est alors à son sommet. Outre le salon de 1902, ces meubles connaissent une large diffusion grâce aux catalogues de vente estampillés Barbedienne, à destination du consommateur.

20. Pour une description exhaustive du salon Jansen aménagé par Simas à l'Exposition universelle de 1900, nous recommandons de se reporter aux deux articles suivants : Gabriel

Mourey, «Décoration moderne », Les Modes, Goupil and Cie, avril 1901 ; Gustave Soulier, « L'ameublement aux Salons », Art et décoration, juillet-décembre 1901, pp. 33-40.

21. Pour plus d'informations sur cet ensemble mobilier, nous préconisons la lecture de l'article «Une salle à manger », Art et décoration, juillet 1902, pp. 167-171; et la consultation des catalogues de vente Maison Barbedienne, $P$-A Dumas successeur, conservés à la bibliothèque des Arts-décoratifs de Paris sous les cotes $\mathrm{Br}$. 736 et $\mathrm{Br}$. 1009. Signalons aussi qu'un buffet bas et une desserte appartenant à cet ensemble sont passés en vente à l'hôtel Drouot le lundi 3 décembre 2012, comme nous l'indique un des catalogues de la société de vente aux enchères Ader Nordmann consacré aux arts décoratifs et sculptures du Xx siècle. 
L'année 1908 annonce une étape nouvelle dans la carrière artistique de E.M. Simas. Après une quinzaine d'années dévolues aux arts industriels, il signe son grand retour dans le domaine du décor de scène. Succédants à Pedro Gailhard, les deux nouveaux directeurs de l'Opéra de Paris, André Messager et Leimistin Broussan, souhaitent apporter "du sang neuf " à cette institution ancestrale. Pour ce faire, ils décident de faire appel à des décorateurs n'ayant encore jamais travaillé pour l'Opéra de Paris. Simas se voit alors sollicité pour le décor du " jardin de Marguerite ", issu de l'opéra Faust de Charles Gounod, d'après la pièce de Goethe. Ce sera le début d'une intense collaboration puisqu'il participera ensuite chaque année, et ce jusqu'en 1915, aux décors de nouvelles pièces pour l'Opéra : la Roussalka, d'après Pouchkine en 1911 ; Roma de Jules Massenet en 1912 ; Fervaal de Vincent d'Indy en 1913 ; Parsifal de Richard Wagner en 1914 ; Mademoiselle de Nantes, ballet sur une chorégraphie de François Ambrosiny en 1915, et enfin, l'Esclarmonde de Jules Massenet en 1923. Parallèlement, il produit des décors pour de nombreux théâtres parisiens tels que le Théâtre national de l'Odéon ${ }^{22}$, l'Olympia ${ }^{23}$, le Théâtre des Champs Élysées ${ }^{24}$, la Comédie Française ${ }^{25}$, l'Apollo $^{26}$, le Théâtre du Gymnase ${ }^{27}$ ainsi que pour la compagnie française du Théâtre Shakespeare ${ }^{28}$.

Les décors qu'il confectionne pour l'Opéra de Paris s'inscrivent dans la droite lignée des créations fastueuses de l'époque romantique qui connaissent leur apogée avec les décorateurs Charles-Antoine Cambon et Edouard Despléchin, puis avec leurs successeurs Jean-Baptiste Lavastre et son associé Eugène Carpezat. Sur le modèle de ses prédécesseurs, les décors que Simas conçoit dans l'atelier qu'il reprend à Carpezat s'appuient sur de minutieuses recherches historiques, topographiques et archéologiques qu'il effectue à la bibliothèque afin de retranscrire le plus fidèlement possible le lieu de l'action de la pièce. Évoquant ces décors plus vrais que nature, un critique contemporain rapporte même que « de vraies fleurs » ainsi qu'un "pommier non moins véritablement fleuri" se sont introduits dans les "jardins de Marguerite ${ }^{29}$. Il faut dire que Simas aime rendre l'impression de vérité. À cet égard, le panneau mouvant qu'il réalise pour l'opéra Parsifal fait sensation : se déroulant et s'enroulant à l'aide de bobines énormes, il donnait au public l'illusion parfaite de suivre le héros dans son ascension sur le mont Salva. Héritier de la longue tradition des décors pittoresques et spectaculaires, le style de Simas évolue malgré tout vers le naturalisme et vers un vérisme qui fait écho aux évolutions scénographiques d'alors, incarnées notamment par le théâtre d'André Antoine. Ses " coloris étranges et vaporeux " ravissent l'œil du spectateur, le plus souvent issu de la bourgeoisie, classe sociale montante sous le Second Empire et qui fréquente assidûment les théâtres et l'opéra. Mais l'arrivée de la Grande Guerre, qui plonge la société française dans le chaos, provoque le recul net de la fréquentation des lieux de spectacle et sonne le glas, dans le même temps, de l'activité de décorateur de Simas.

\footnotetext{
22. Au Théâtre national de I'Odéon, Eugène Martial Simas réalise deux décors (un grand salon $\mathrm{d}^{\prime} u n e$ vieille maison du $\mathrm{XvI}^{\mathrm{e}}$ siècle et un bureau-bibliothèque) pour la pièce La maison des juges, de Gaston Leroux, jouée en 1906.

23. Il produit le décor pour Enfin... une revue! de MM. Moreau, Ardot et Albert Laroche, à I'Olympia, en 1912.

24. Eugène Martial Simas élabore le décor de Benvenuto Cellini, opéra d'Hector Berlioz au Théâtre des Champs Elysées, en 1913.

25. Pour la Comédie Française, Simas conçoit le décor de La Furie en 1909, du Ménage de Molière en 1912, comédie en cinq actes et six tableaux de Maurice Donnay, ainsi que de Riquet à la houppe de Théodore de Banville en 1913, en collaboration avec le décorateur Alfred Devred. 26. En 1915, il confectionne le décor de La cocarde de Mimi Pinson, opérette en trois actes de M. Ordonneau et F. Gally, jouée à I'Apollo, sur une musique de M. Goublier fils.

27. Eugène Martial Simas réalise l'intégralité du décor de la pièce de théâtre Petite Reine, au Théâtre du Gymnase, en 1917.

28. En 1910, il conçoit les décors de onze pièces pour la compagnie française du Théâtre Shakespeare fondée par Camille de Sainte-Croix : Comme il vous plaira, Conte d'hiver, La Tempête, Troïlus et Cressida, École de la Pie Grièche, Cymbeline, Les Joyeuses Commères de Windsor, Le songe d'une nuit d'été, Beaucoup de bruit pour rien, Peines d'Amour perdues et Le Marchand de Venise.

29. Chassaigne de Néronde, «La nouvelle mise en scène de Faust à I'Opéra », Les annales politiques et littéraires, 2 février 1908, pp. 108,109.
} 


\section{Du décor monumental au petit format}

Le dernier tiers de la carrière d'Eugène Martial Simas est occupé par l'illustration d'ouvrages, et plus particulièrement de poèmes, ainsi que par la peinture de chevalet. Déjà quelques années plus tôt, il s'était essayé à l'art de l'affiche, domaine qui avait pris une ampleur nouvelle à partir des années 1850 , grâce au perfectionnement de la technique de la gravure en couleur, aussi appelée chromolithographie. Le Musée des Arts décoratifs de Paris conserve l'une de ses affiches (fig. 5). À visée publicitaire, elle encourage à la consommation de la "Ludivine ", liqueur fabriquée à l'abbaye de Poissy, ville qui vit grandir l'artiste.
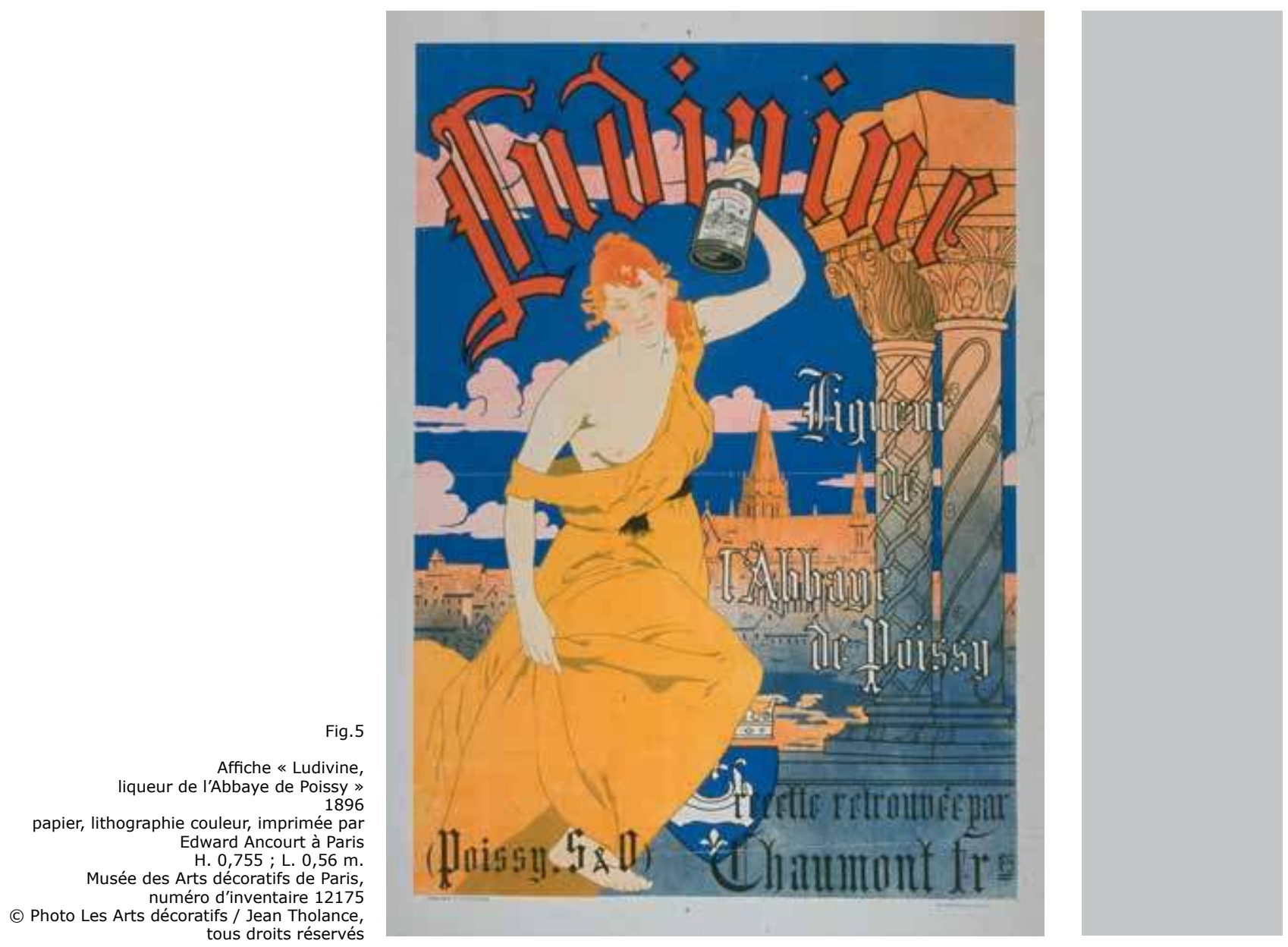

Une autre, conservée aux cabinets des estampes de la BnF, nous annonce la soirée du bal des étudiants se déroulant au Grand-Théâtre de Lyon, en 1893. Deux ans plus tard, l'illustration d'un calendrier de l'année 1895, publié en anglais et relatant les pérégrinations au fil des mois d'un couple de jeunes bourgeois, rend compte de la diversité des supports que Simas plébiscitait alors, ainsi que de leur diffusion au-delà des frontières françaises.

En 1904 et 1905, il est appelé à décorer un nombre choisi de fables de Jean de La Fontaine pour Le Figaro illustré. Dans des tons pastel, les illustrations qu'il élabore selon une vision très personnelle complètent discrètement le texte sans le paraphraser. On retrouve là tout son talent de décorateur qui, de la même manière qu'au théâtre, prend soin de donner un cadre scénique à l'action sans pour autant l'écraser et l'ensevelir sous le faste. Il poursuit en 1921 avec l'illustration du frontispice d'un recueil d'études de Dante conçu pour le sixième centenaire de la mort du poète. Souvenons-nous que ce dernier fut une source d'inspiration intarissable pour les artistes du XIx siècle. De Delacroix à Blake en passant par Cabanel, Bouguereau et Rodin, tous puisèrent dans sa Divine Comédie les sentiments les plus sombres qui animent l'âme humaine pour façonner un art tourmenté et passionné. A contrario, l'image que donne Simas 
du poète, fortement inspirée d'un portrait qu'en fit Sandro Botticelli, est celle d'un homme sage, aux traits mûrs, apparaissant dans toute sa grandeur classique. Cette aspiration à un art objectif et impassible n'a rien d'étonnant chez Simas. En effet, ses œuvres ont des affinités certaines avec le mouvement littéraire du Parnasse dont les membres prônaient, en réaction contre les excès lyriques du romantisme, une poésie dépassionnée et descriptive. Il est d'ailleurs à noter que le portrait de Dante qu'il élabore est directement suivi des vers majestueux du poète parnassien Pierre de Nolhac (1859-1936). Simas alliera également ses talents d'illustrateurs avec un autre membre du Parnasse, Édouard Beaufils (1868-1941), dont il ornera en 1929 le recueil de poèmes Le Sortilège, couronné la même année par l'Académie française. Dans un style dépouillé évoquant quasiment un travail d'esquisse, Simas choisit de se servir uniquement d'encre noire pour signifier l'Italie éternelle des poèmes de Beaufils.

Quant à la peinture de chevalet, si elle occupe exclusivement la fin de sa carrière, elle a toutefois accompagné l'artiste toute sa vie. Dès l'âge de 24 ans, il expose à Versailles puis au Salon de la société des artistes français avant de devenir associé et enfin d'être nommé sociétaire de la Société nationale des beaux$\operatorname{arts}^{30}$. Le plus souvent, il dépeint les paysages qu'il observe lors de ses voyages en Normandie $^{31}$, en Italie ${ }^{32}$ ou dans le sud-ouest ${ }^{33}$ de la France. On sait notamment qu'il rapporte des Balkans cinquante-six toiles qui seront exposées en 1919 à la célèbre galerie Georges Petit. Mais Simas se plait aussi à réaliser les portraits de ses amis artistes tels que l'architecte Albert-Désir Guilbert (1866-1949) ou encore le peintre Germain David-Nillet (1861-1932). Bien que cette activité ne constitue pas l'essentiel de son travail, elle lui permet toutefois de conserver une visibilité grâce à l'exposition de ses tableaux dans les Salons.

\section{Simas, un artiste resté méconnu}

Eugène Martial Simas disparaît le 30 septembre 1939 à l'âge de 77 ans alors qu'il rend visite à un ami en Bourgogne ${ }^{34}$. Sa mort passe relativement inaperçue, ce qui s'explique probablement par l'actualité politique du moment: l'entrée en guerre de la France contre l'Allemagne. Aussi, en ces temps de troubles, les journaux de l'époque communiquent exclusivement les faits de guerre et aucun papier rétrospectif sur la vie et la carrière de l'artiste ne voit le jour. Il faut ajouter qu'Eugène Martial Simas n'a pas engendré de descendance qui aurait pu conserver les archives personnelles de l'artiste, sources précieuses de renseignements. Corollairement, l'inventaire après décès de ses biens, dressé généralement à la demande des héritiers potentiels, n'a pas eu lieu. Enfin, avec le temps, certains projets auxquels il participa furent démolis comme le jardin d'hiver de la maison des Clermont-Tonnerre à Maisons-Laffitte, en banlieue parisienne, édifice que l'on devait à Louis Granet (1852-1935) ${ }^{35}$ et dont les panneaux de faïence furent en partie réutilisés pour décorer un restaurant parisien ${ }^{36}$.

30. Eugène Martial Simas est nommé sociétaire de la Société nationale des beaux-arts en 1924, à l'âge de 62 ans.

31. De Normandie, il rapporte Le calvaire de Bénouville, Le carrefour de Ranville, La divette à Cabourg, Marée Montante, coucher de soleil, Le Soir (vieux Cabourg), Les dunes à Cabourg, Mer basse à Cabourg et Pêcheurs de Dives, toiles qu'il expose entre 1894 à 1905.

32. En 1922, il expose au salon de la Société nationale des beaux-arts L'église du Monte dei Cappuccini à Turin.

33. Inspiré par les paysages du sud-ouest de la France, Simas peint les tableaux « La dépiquaison » à Pescadoure, L'Arize à Montesquieu-Volvestre, L'automne à Montesquieu-Volvestre, exposés en 1920, et Un châtaigner de l'Aveyron, Un moulin, vallée de l'Aveyron, exposés en 1925.

34. Registres de décès de la mairie de Saint-Julien-du-Sault, Yonne, Bourgogne : Acte de décès d’Eugène Martial Simas, réalisé le 30 septembre 1939, à Saint-Julien-du-Sault : « Le trente septembre mil neuf cent trente-neuf, onze heures, est décédé Eugène Martial Simas, né à Paris le trente et un août mil huit cent soixante-deux, artiste peintre, domicilié 4 rue Armand Carrel, [...] époux de Anne Marie Dalbergue. Dressé le trente septembre mil neuf cent trente-neuf, dix-huit heures, sur la déclaration de Eugène Coltat, carrossier, soixante-deux ans, domicilié à Saint-Julien-du-Sault. »

35. Une photographie de la décoration intérieure du jardin d'hiver de la villa des ClermontTonnerre à Maisons-Laffitte est conservée à la Cité de l'Architecture et du Patrimoine, au sein du fonds Granet, André (1881-1974), Louis (1852-1935), Bernard (1925-1981), sous la cote 086 ifa.

36. Aujourd'hui, les panneaux de carreaux de faïence de Sarreguemines, dont on doit les modèles à Simas et qui décoraient autrefois le jardin d'hiver, ornent le perron du restaurant La Fermette Marbeuf, situé dans le $8^{\mathrm{e}}$ arrondissement de Paris. 


\section{Et aujourd'hui ?}

Bien que des zones d'ombre subsistent encore sur la composition de son œuvre, les éléments qui nous sont parvenus permettent d'affirmer que Simas est un décorateur incontournable qui a marqué son époque. Véritable orchestrateur de projets décoratifs, il a conçu un art au service des gens, qui se mêle à leur vie quotidienne, marquant l'environnement urbain de son empreinte. Son univers, bien loin de l'esthétique décadentiste fin-de-siècle et du mysticisme prôné par les symbolistes, est celui d'un monde idéalisé et pittoresque, délicat et raffiné qui trouve davantage de connivences avec le mouvement parnassien, dont il admirait la poésie. Les motifs et les thèmes qu'il développe - la flore, la Femme - renvoient à l'Art nouveau mais également au Japonisme, tendance de la fin du XIx ${ }^{e}$ siècle qui inspira de nombreux artistes comme Édouard Manet ou les peintres Nabis.

Témoignage artistique unique de la Belle Époque, l'œuvre d'Eugène Martial Simas ne doit pas être négligée mais au contraire, préservée. C’est le cri d'alarme que lance l'association Patrimoine-Environnement, militant pour la protection du patrimoine de France et porteuse du projet de restauration des panneaux de faïence de la gare de Tours. En effet, ayant subi les outrages du temps et la pollution aérienne de la gare, le support d'origine des tableaux, fait de chaux, est à ce jour fortement dégradé et n'assure plus l'adhérence des carreaux de céramique peinte, qui de ce fait se décollent et menacent de tomber et de détruire les compositions. Par ailleurs, la suie produite par les locomotives sous les grandes halles voyageurs pendant près d'un siècle, a fortement encrassé les carreaux de céramique. Aussi, dans l'optique de leur indispensable restauration, un appel aux dons a été lancé sur le site de financement participatif ulule.com. Espérons qu’il porte ses fruits afin de rendre à ces délicieuses compositions l'éclat et la postérité qu'elles méritent.

\section{L'auteur}

Après une licence d'histoire de l'art à l'Université de Bourgogne, Constance Desanti a obtenu le diplôme de muséologie puis celui de $2^{\mathrm{e}}$ cycle de l'École du Louvre. En 2013, elle a rédigé un mémoire de Recherche sous la direction d'Alice Thomine-Berrada et d'Yves Badetz autour de la personne et de l'œuvre d'Eugène Martial Simas (1862-1939). 